\title{
Morphological Characters, Occurrence and Distribution among Members of the Family Solanaceae in Parts of the Niger Delta Ecological Zone
}

\author{
${ }^{* 1}$ WAHUA, C; EDWIN-WOSU, NL \\ Department of Plant Science and Biotechnology, \\ University of Port Harcourt, Choba, \\ P.M.B.5323, Nigeria. \\ *Email:nhechinemenum@yahoo.com;Email:nsirim.edwin-wosu@uniport.edu.ng
}

\begin{abstract}
This study investigated a comparative micro-morphological features of 14 species in the family Solanaceae, using trichome and stomatal complements in delimitation of species and genera within the taxon, family. The genus Solanum L. is the largest among the genera in Solanaceae. Solanum aethiopicum Linn. Solanum macrocarpon Linn. Solanum torvum Swartz. Solanum anomalum Thonn. Solanum erianthum D. Don, are covered with stellate trichomes. While Solanum nigrum Linn., Solanum lycopersicum Linn., Solanum incanum Linn., Datura spp., Schwenckia spp., Capsicum spp., Physalis spp. have simple uniseriate trichomes. S. aethiopicum Linn. S. torvum Swartz. S. lycopersicum Linn, and Physalis micrantha Linn, revealed higher trichome indices. In most species, these trichomes are not visibly observed with the naked eyes. While in some others such as in $S$. torvum and $S$. lycopersicum the trichomes are observable on the plants. Glandular hairs are also present in Solanaceae. Stomata is amphistomatic, types commonly observed are: anisocytic and anomocytic, while paracytic and tetracytic stomata are sometimes revealed in stem epidermis in Solanaceae. The usefulness of trichome and stomatal complements in species and generic taxa are recommended as pertinent characters for classification in Solanaceae and their density may vary from one ecological zone to another. OJASEM

http://dx.doi.org/10.4314/jasem.v20i3.12
\end{abstract}

Key words: Solanaceae, Solanum, Stomata, trichomes, Complements, Comparative.

\section{INTRODUCTION}

Stomata are considered to be one of the major epidermal structures within the leaf organ that have allowed higher plants to adapt to virtually all terrestrial environments on earth, this is made possible by means of adjustment of their size, density and distribution (Zarinkamar, 2006b). An alteration of leaf stomatal density can be used as an indicator of environmental change (Case, 2004). Solanum roustrum, the shrubby nightshade, is a thorny perennial with velvet leaves and stems due to dense stellate trichomes present on all over the plant (USDA, 2015). Solanaceae is predominated mostly by anomocytic stomata. Trichome and stomatal complements are necessary diagnostic characters used in identification at species and generic levels. The type of hair can be of diagnostic value at species level, sometimes also at generic level, but rarely at family level (Cutler, 1977).The word 'uniseriate' is really an anatomical term rather than morphological and does not describe the shape. 'Multiseriate' is not unique to trichomes, it could also mean multi layers as in epidermal and hypodermal axial parenchyma (Metcalfe and Chalk, 1979).
The present study is aimed at producing a more current and comprehensive taxonomic data involving the micro-morphological characters, their occurrence and distribution among members of the family Solanaceae.

\section{MATERIALS AND METHODS}

Study Area: The areas covered were different Localities in Rivers State, Imo State and Abia State parts of the Niger Delta, Nigeria.

Sample Collection: The materials used for this study were collected from both cultivated domestic species and wild species or raised from seeds purchased from the fruit and vegetable markets.

Sample Preparation: Fresh leaves and stems were peeled and bleached using sodium hypochlorite for about 2 minutes following the method of Cutler (1978). For other fresh leaves whose epidermal layers were difficult to peel, chemical frictions or scraping method were used, usually the surface to be examined was placed on a glass slide while the other surface was carefully cleared by flooding with $5 \%$ sodium hypochlorite and scraping with cork material 
or razor. The cleared epidermal layers obtained were then washed in several changes of distilled water and stained with Alcian blue or safranin and temporarily mounted in aqueous glycerol solution (Cutler, 1978). Photomicrographs were taken from good preparations. Stomatal studies were done from the cleared leaves and stems. The length and width of the stomatal complexes (guard cells) were measured using a calibrated eye piece graticule following the method of Arnold (1973). The stomata observed were viewed with the light microscope and were measured or calculated in unit area using the stomatal index [S.I.] formula as shown below:

S.I. $=\frac{S}{E+S} \times \frac{100}{1}$, equation 1.

\section{RESULT AND DISCUSSION}

Stellate or star-shaped trichomes are observed in plates $1 \mathrm{a}, 1 \mathrm{~b}, 2 \mathrm{a}, 2 \mathrm{~b}$, figures $1 \mathrm{a}$ to $1 \mathrm{p}$ and figures $2 \mathrm{a}$ to $2 \mathrm{~d}$ as observed in S. aethiopicum Linn. S. torvum Swartz. S. anomalum Thonn. S. erianthum D. Don. respectively. While the simple uniseriate trichomes aled in plates $3 \mathrm{a}$ to $8 \mathrm{c}$ and figures $2 \mathrm{e}$ to $3 \mathrm{r}$ respectively. The studies further revealed the presence of anomocytic, anisocytic, tetracytic, paracytic, stomata. S. nigrum is observed with the
Where $S$ and $E$ mean numbers of stomata and epidermal cells within the particular area under investigation.

The same formula was applicable for the calculation of trichome index (T.I.), in this case, trichome (T) was used instead of stomata: T.I. $=\frac{T}{E+T} \times \frac{100}{1}$, equation 2.

Statistical Analysis: The mean \pm standard deviation for trichome and stomatal characteristics of Solanaceae studied was done.

are revealed in the genera: Solanum (S. nigrum Linn. S. incanum Linn. S. lycopersicum Linn.), Datura, Schwenckia, Physalis, Capsicum etc. The simple or non-stellate trichomes are more universally distributed in Solanaceae than the stellate types. The simple uniseriate and multiseriate trichomes are reve highest stomatal index among the species studied. (Table 1). S. aethiopicum, S. torvum, S. lycopersicum and Physalis micrantha showed higher trichome indices than others. (Table 2)
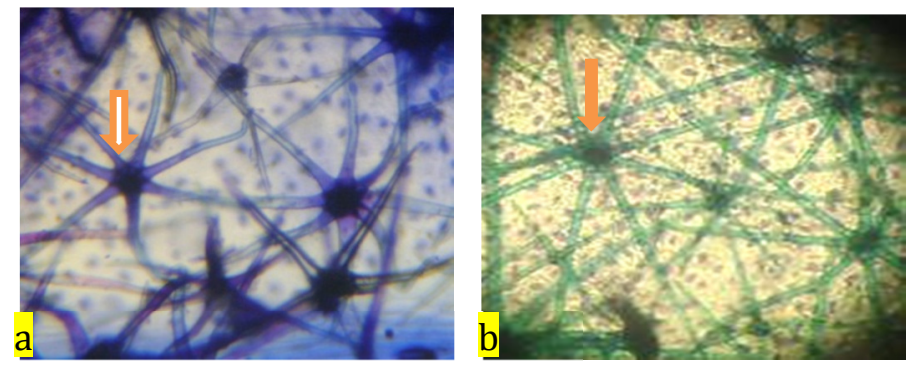

Arrows revealed stellate trichomes

Plates 1a: S. aethiopicum Linn. Adaxial foliar epidermis with numerous stellate trichomes. 1b: S. torvum Swartz. Adaxial foliar epidermis with high density of stellate trichomes. Morphologically, most members of the genus 'Solanum' with rough lamellae possess stellate trichomes, it is envisaged that the numerous dense nature of the hairs is the reason for the leaf texture, as also supported by (USDA, 2015).
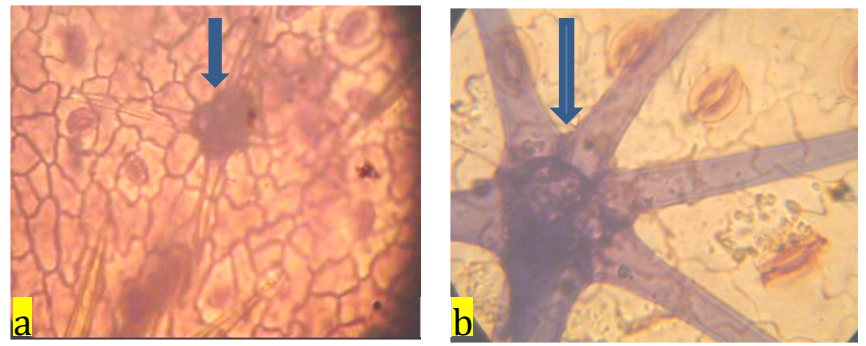
Plate 2a: S. torvum Swartz. Abaxial foliar epidermis with less trichome density. 2b: S. macrocarpon Linn. abaxial foliar epidermis with 6 branched stellate trichome. Arrows revealed stellate trichomes.

Solanum species observed with stellate trichomes possessed basically anisocytic and anomocytic stomata. The number of the branches may differ within a given species, (See figures1 and 2a to 2d). There were varying degrees of stomatal and trichome densities on the adaxial and abaxial foliar surfaces of the species.
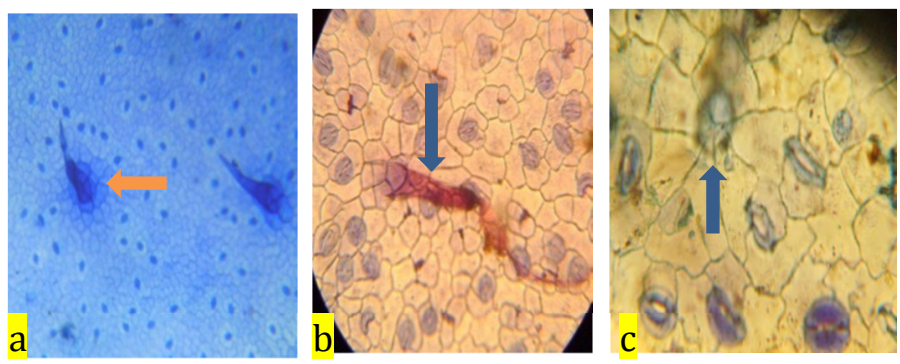

Plate 3a: S. nigrum Linn. abaxial foliar epidermis possessed spine-like uniseriate trichomes, (figure 2e). 3b: Datura metel Linn. Adaxial foliar epidermis revealed blunt-end uniseriate trichomes. 3c: Datura metel Linn. abaxial foliar epidermis. Black arrow showed glandular trichome associated with oil gland. (Figures 2f; 3b; 3e and 31).
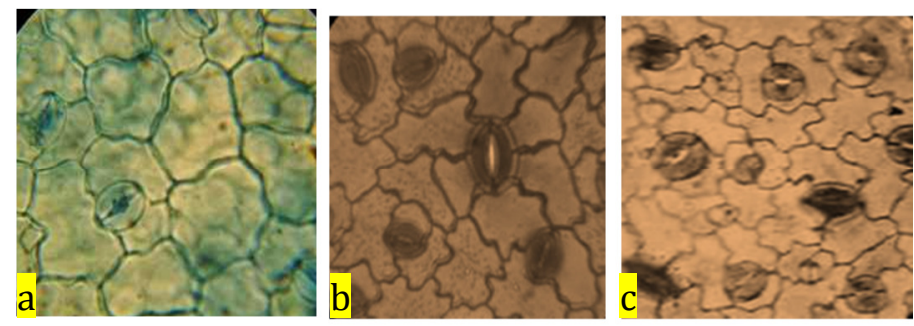

Plate 4a: S. incanum Linn. Adaxial foliar epidermis. 4b: S. incanum Linn. Abaxial foliar epidermis with anomocytic and anisocytic stomata, 4c: S. lycopersicum Linn. Abaxial foliar epidermis. S. incanum Linn. And S. lycopersicum Linn. are tomentosed with uniseriate trichomes. (See figures $2 \mathrm{i}, 2 \mathrm{~g}, 3 \mathrm{~g}, 3 \mathrm{~m}$ and $3 \mathrm{p}$ ).
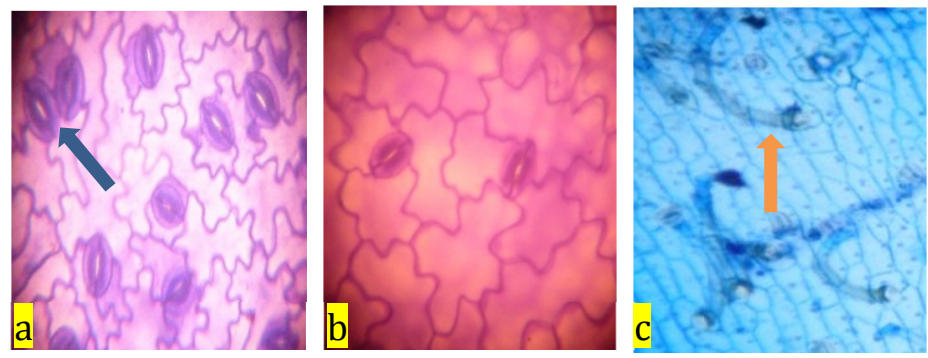

Plate 5a: Schwenckia americana Linn. Abaxial foliar epidermis. Black arrow revealed contiguous cells. 5b: Schwenckia americana Linn. Adaxial foliar epidermis. 5c: Schwenckia americana Linn. Stem epidermis showcased anomocytic stomata. White arrow showed curved hair with bulge head termed covering trichome, which signified a kind of oil secretory function which gave the leaves and stems glossary outlook. (Figure $2 \mathrm{q}$ ). The presence of tapering trichomes was also discovered in Schwenckia, (see figure 3g).
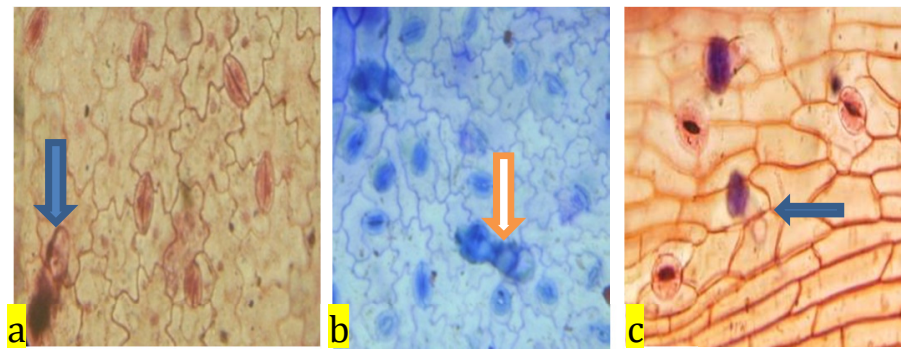

WAHUA, $C ; E D W I N-W O S U, N L$ 
Plate 6a: Physalis angulata Linn. Adaxial foliar epidermis. Arrow revealed uniseriate hair. Plate 6b: Physalis angulata Linn. Abaxial foliar epidermis. Arrow showed tricellular glandular trichome with bulge ends. (Figures $2 \mathrm{~h}$ and 3v). Plate 6c: Physalis angulata Linn. Stem epidermis. Arrow revealed biseriate trichome. (Figures 2g, 2j, 2r, 3d, 3o, 3q and 3u) commonly found in Physalis spp.
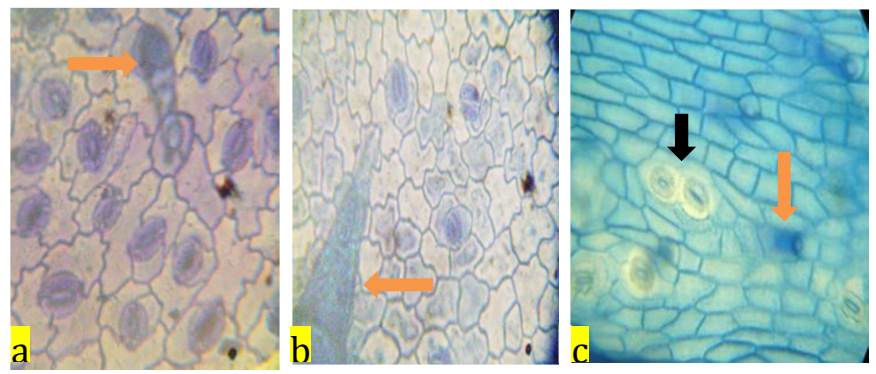

Plate 7a: Capsicum frutescens Linn. Abaxial foliar epidermis, arrow revealed clavate trichome. (See figures $2 \mathrm{~g}$, $2 \mathrm{~m}$ and 2r). Plate 7b: Capsicum frutescens Linn. Adaxial foliar epidermis with conical trichome. Plate 7c: Capsicum frutescens Linn. Stem epidermis. (Figure 2p) Black arrow showed contiguous cells (see figure 4c), while the brown arrow indicated glandular trichome believed to contain capsaicin.
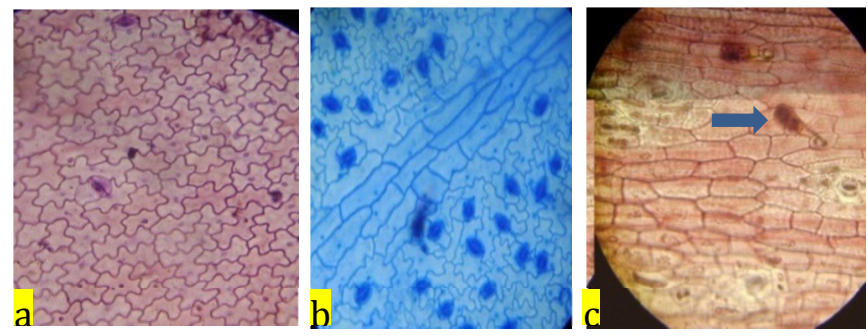

Plate 8a: Capsicum annuиm Linn Adaxial foliar epidermis. 8b: Capsicum annuиm Linn Abaxial foliar epidermis. 8c: Capsicum апnиит Linn Stem epidermis. Arrow showed biseriate clavate trichome. (See Figures: $3 \mathrm{c}, 3 \mathrm{f}, 3 \mathrm{j}, 3 \mathrm{k}, 3 \mathrm{r}$ and $3 \mathrm{~s})$. The average number of stomata in the lower epidermis is more numerous than as observed in the upper epidermis.

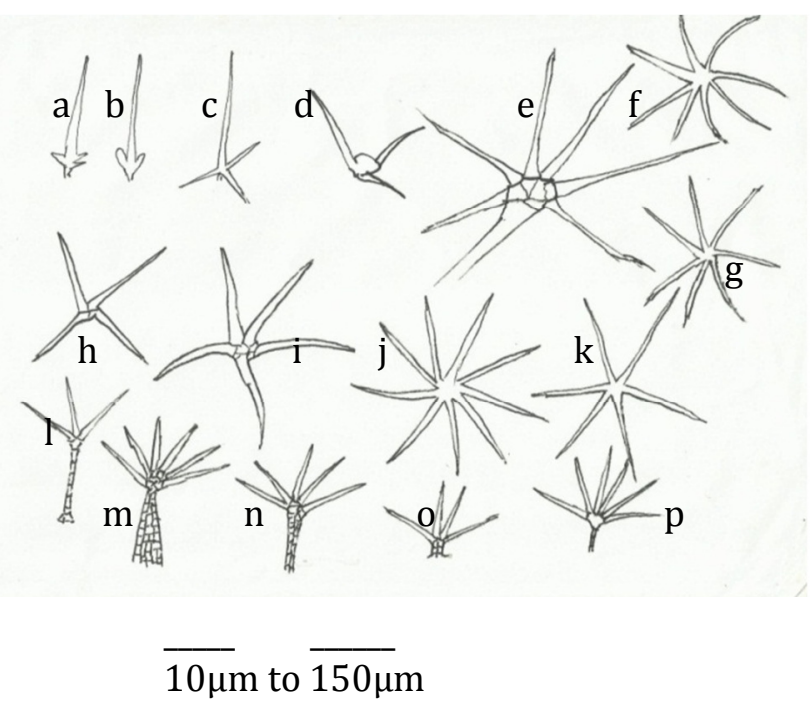

Figure 1: Stellate trichomes in Solanaceae. 1a to $1 \mathrm{~d}$ are prominently present during the early growth stage or primary phase of development observed with two (2), three (3) or Four (4) short miniature branched stellates. 
While 1e to $1 \mathrm{p}$ revealed stellate trichomes found regularly during secondary growth phase. Figures $1 \mathrm{~m}$ to $1 \mathrm{o}$ showed multiseriate stalked stellate trichomes.

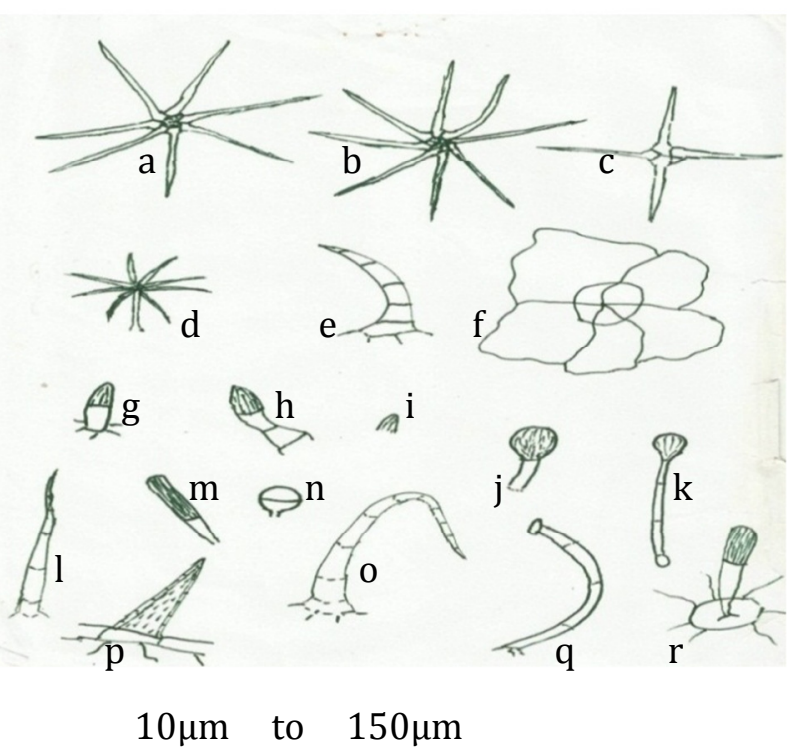

Figure 2: Stellate trichomes in Solanaceae.2a to 2d. While 2e revealed Spine-like trichome in S. nigrum Linn. 2f: Glandular trichome in Solanaceae. $2 \mathrm{~g}$ to 2r: Simple uniseriate trichomes in Solanaceae.

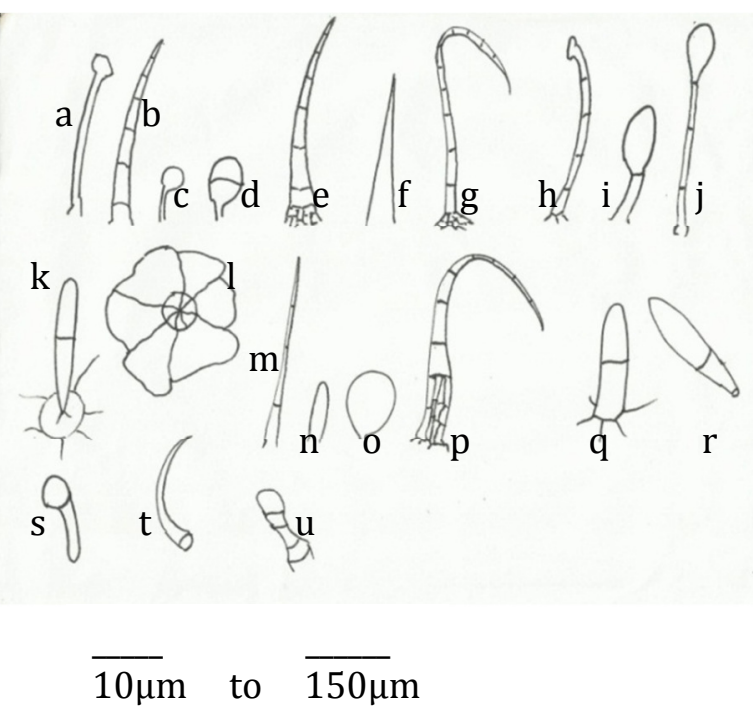

Fig 3: Simple uniseriate trichome complements in Solanaceae. $3 \mathrm{a}$ and $3 \mathrm{~h}$ are covering trichomes unique to Schwenckia americana along with $3 \mathrm{~g}$ which is a tapering trichome, $3 \mathrm{~b} ; 3 \mathrm{e}$, and 31 were observed in Datura metel. $3 \mathrm{c}, 3 \mathrm{f}, 3 \mathrm{j}, 3 \mathrm{k}, 3 \mathrm{r}$ and $3 \mathrm{~s}$ were revealed in Capsicum spp., $3 \mathrm{~g}, 3 \mathrm{~m}$ and $3 \mathrm{p}$ showed in Solanum lycopersicum, while 3d, 3o, 3q and 3u were found present in Physalis species. 


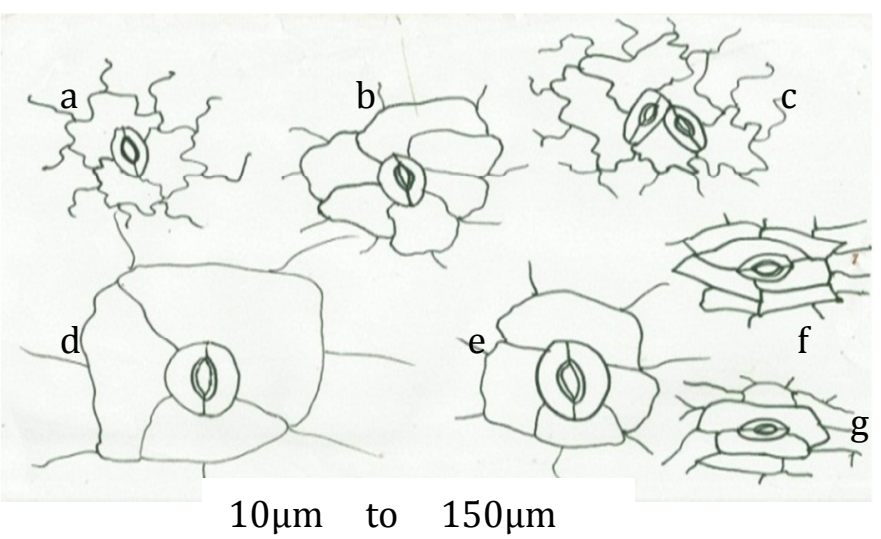

Fig 4: Stomatal types in Solanaceae. 4a to 4b: Anomcytic; 4c: contiguous cells; 4d to 4e: anisocytic; 4f: anomocytic; 4g: paracytic stoma. Paracytic stomata observed in stem epidermis but not as common as anomocytic and anisocytic stomata.

Table 1: Stomatal Indices of some Members of Solanaceae within the Niger Delta Eco zone, Nigeria

\begin{tabular}{|c|c|c|c|c|c|c|c|c|c|}
\hline \multirow{2}{*}{$\begin{array}{l}\text { UPH } \\
\text { Reference } \\
\text { No } \\
\end{array}$} & \multirow[t]{2}{*}{ Solanaceae } & \multicolumn{4}{|c|}{ Adaxial( Upper) Foliar Surface } & \multicolumn{4}{|c|}{ Abaxial(Lower) Foliar Surface } \\
\hline & & $\begin{array}{l}\text { No of } \\
\text { Stomatal } \\
\text { Cells }\end{array}$ & $\begin{array}{l}\text { No of } \\
\text { Epiderm } \\
\text { al Cells }\end{array}$ & $\begin{array}{l}\text { Stomatal } \\
\text { Index (SI) }\end{array}$ & $\begin{array}{l}\text { Stomatal } \\
\text { Type }\end{array}$ & $\begin{array}{l}\text { No of } \\
\text { Stomatal } \\
\text { Cells }\end{array}$ & $\begin{array}{l}\text { No of } \\
\text { Epidermal } \\
\text { Cells }\end{array}$ & $\begin{array}{l}\text { Stomatal } \\
\text { Index (SI) }\end{array}$ & $\begin{array}{l}\text { Stomatal } \\
\text { Type }\end{array}$ \\
\hline CWOO1 & $\begin{array}{l}\text { Solanum } \\
\text { aethiopicum } \\
\text { Linn. }\end{array}$ & 45 & 155 & $22.50 \%$ & Anisocytic & 75 & 235 & $24.19 \%$ & Anisocytic \\
\hline CWOO2 & $\begin{array}{l}\text { Solanum } \\
\text { nigrum Linn. }\end{array}$ & 47 & 105 & $30.92 \%$ & Anisocytic & 96 & 409 & $19.01 \%$ & Anisocytic \\
\hline CWOO3 & $\begin{array}{l}\text { Solanum } \\
\text { torvum } \mathrm{Sw} .\end{array}$ & 30 & 212 & $12.40 \%$ & Anisocytic & 38 & 168 & $18.45 \%$ & Anisocytic \\
\hline CWOO4 & $\begin{array}{l}\text { Solanum } \\
\text { macrocarpon } \\
\text { Linn. }\end{array}$ & 67 & 221 & $23.26 \%$ & Anisocytic & 90 & 370 & $19.57 \%$ & Anisocytic \\
\hline CWOO5 & $\begin{array}{l}\text { Solanum } \\
\text { anomalum } \\
\text { Thonn. }\end{array}$ & 60 & 242 & $19.86 \%$ & Anisocytic & 90 & 370 & $19.57 \%$ & Anisocytic \\
\hline CWOO6 & $\begin{array}{l}\text { Solanum } \\
\text { incanum Linn. }\end{array}$ & 98 & 305 & $24.32 \%$ & Anisocytic & 98 & 305 & $24.32 \%$ & Anisocytic \\
\hline CWOO7 & $\begin{array}{l}\text { Solanum } \\
\text { erianthum D. } \\
\text {.Don. }\end{array}$ & 70 & 295 & $19.18 \%$ & Anisocytic & 98 & 295 & $24.94 \%$ & Anisocytic \\
\hline CWOO8 & $\begin{array}{l}\text { Solanum } \\
\text { lycopersicum } \\
\text { Linn. }\end{array}$ & 55 & 230 & $19.30 \%$ & Anisocytic & 44 & 180 & $19.64 \%$ & Anisocytic \\
\hline CWO9 & $\begin{array}{l}\text { Datura metel } \\
\text { Linn. }\end{array}$ & 30 & 103 & $22.56 \%$ & Anisocytic & 15 & 80 & $15.79 \%$ & Anisocytic \\
\hline CWO10 & $\begin{array}{l}\text { Schwenckia } \\
\text { americana } \\
\text { Linn. }\end{array}$ & 3 & 45 & $6.25 \%$ & $\begin{array}{l}\text { Anomocyti } \\
\text { c }\end{array}$ & 15 & 45 & $25.00 \%$ & $\begin{array}{l}\text { Anomocyti } \\
\text { c }\end{array}$ \\
\hline CWO11 & $\begin{array}{l}\text { Physalis } \\
\text { angulata } \\
\text { Linn. }\end{array}$ & 15 & 95 & $13.64 \%$ & $\begin{array}{l}\text { Anomocyti } \\
\text { c }\end{array}$ & 20 & 80 & $20.00 \%$ & $\begin{array}{l}\text { Anomocyti } \\
\text { c }\end{array}$ \\
\hline CW012 & $\begin{array}{l}\text { Physalis } \\
\text { micrantha } \\
\text { Linn. }\end{array}$ & 18 & 98 & $15.52 \%$ & $\begin{array}{l}\text { Anomocyti } \\
\text { c }\end{array}$ & 25 & 96 & $20.66 \%$ & $\begin{array}{l}\text { Anomocyti } \\
\text { c }\end{array}$ \\
\hline CWO13 & $\begin{array}{l}\text { Capsicum } \\
\text { frutescens } \\
\text { Linn. }\end{array}$ & 5 & 78 & $6.02 \%$ & $\begin{array}{l}\text { Anomocyti } \\
\text { c }\end{array}$ & 18 & 118 & $13.24 \%$ & $\begin{array}{l}\text { Anomocyti } \\
\text { c }\end{array}$ \\
\hline CWO14 & $\begin{array}{l}\text { Capsicum } \\
\text { aпnиum Linn. }\end{array}$ & 5 & 78 & $6.02 \%$ & $\begin{array}{l}\text { Anomocyti } \\
\text { c }\end{array}$ & 41 & 117 & $25.95 \%$ & $\begin{array}{l}\text { Anomocyti } \\
\text { c }\end{array}$ \\
\hline
\end{tabular}


Table 2: Trichome Indices of some Members of Solanaceae within the Niger Delta Eco zone, Nigeria

\begin{tabular}{|c|c|c|c|c|c|c|c|c|c|}
\hline \multirow{2}{*}{$\begin{array}{l}\text { UPH } \\
\text { Reference } \\
\text { No } \\
\end{array}$} & \multirow[t]{2}{*}{ Solanaceae } & \multicolumn{4}{|c|}{ Adaxial( Upper) Foliar Surface } & \multicolumn{4}{|c|}{ Abaxial(Lower) Foliar Surface } \\
\hline & & $\begin{array}{l}\text { No of } \\
\text { Trichomes } \\
\text { Cells }\end{array}$ & $\begin{array}{l}\text { No of } \\
\text { Epidermal } \\
\text { Cells }\end{array}$ & $\begin{array}{l}\text { Trichome } \\
\text { Index (SI) }\end{array}$ & $\begin{array}{l}\text { Trichome } \\
\text { Type }\end{array}$ & $\begin{array}{l}\text { No of } \\
\text { Trichomes } \\
\text { Cells }\end{array}$ & $\begin{array}{l}\text { No of } \\
\text { Epidermal } \\
\text { Cells }\end{array}$ & $\begin{array}{l}\text { Tirchome } \\
\text { Index (SI) }\end{array}$ & $\begin{array}{l}\text { Trichome } \\
\text { Type }\end{array}$ \\
\hline CWOO1 & $\begin{array}{l}\text { Solanum } \\
\text { aethiopicum } \\
\text { Linn. } \\
\end{array}$ & 155 & 325 & $32.29 \%$ & Stellate & 125 & 330 & $27.47 \%$ & Stellate \\
\hline CWOO2 & $\begin{array}{l}\text { Solanum nigrum } \\
\text { Linn. }\end{array}$ & 5 & 250 & $01.96 \%$ & Uniseriate & 96 & 545 & $15.97 \%$ & Uniseriate \\
\hline CWOO3 & $\begin{array}{l}\text { Solanum torvum } \\
\text { Sw. }\end{array}$ & 115 & 309 & $27.12 \%$ & Stellate & 85 & 301 & $22.02 \%$ & Stellate \\
\hline CWOO4 & $\begin{array}{l}\text { Solanum } \\
\text { macrocarpon } \\
\text { Linn. }\end{array}$ & 39 & 285 & $12.04 \%$ & Stellate & 31 & 291 & $09.63 \%$ & Stellate \\
\hline CWOO5 & $\begin{array}{l}\text { Solanum } \\
\text { anomalum } \\
\text { Thonn. }\end{array}$ & 25 & 302 & $07.65 \%$ & Stellate & 20 & 425 & $04.49 \%$ & Stellate \\
\hline CWOO6 & $\begin{array}{l}\text { Solanum } \\
\text { incanum Linn. }\end{array}$ & 10 & 410 & $02.38 \%$ & Uniseriate & 08 & 420 & $01.87 \%$ & Uniseriate \\
\hline CWOO7 & $\begin{array}{l}\begin{array}{l}\text { Solanum } \\
\text { erianthum } \\
\text {.Don. }\end{array} \\
\end{array}$ & 20 & 390 & $04.88 \%$ & Stellate & 28 & 382 & $06.83 \%$ & Stellate \\
\hline CWOO8 & $\begin{array}{l}\text { Solanum } \\
\text { lycopersicum } \\
\text { Linn. } \\
\end{array}$ & 115 & 308 & $27.19 \%$ & Uniseriate & 70 & 290 & $19.44 \%$ & Uniseriate \\
\hline CWO09 & $\begin{array}{l}\text { Datura metel } \\
\text { Linn. }\end{array}$ & 10 & 350 & $02.94 \%$ & Uniseriate & 15 & 250 & $05.66 \%$ & Uniseriate \\
\hline CWO10 & $\begin{array}{l}\text { Schwenckia } \\
\text { americana Linn. }\end{array}$ & 30 & 300 & $09.09 \%$ & Uniseriate & 30 & 320 & $08.57 \%$ & Uniseriate \\
\hline CWO11 & $\begin{array}{l}\text { Physalis } \\
\text { angulata Linn. }\end{array}$ & 3 & 250 & $01.19 \%$ & Uniseriate & 4 & 300 & $01.32 \%$ & Uniseriate \\
\hline CW012 & $\begin{array}{l}\text { Physalis } \\
\text { micrantha Linn. }\end{array}$ & 51 & 400 & $11.31 \%$ & Uniseriate & 203 & 530 & $27.69 \%$ & Uniseriate \\
\hline CWO13 & $\begin{array}{l}\text { Capsicum } \\
\text { frutescens } \text { Linn. }\end{array}$ & 10 & 325 & $02.99 \%$ & Uniseriate & 30 & 335 & $08.22 \%$ & Uniseriate \\
\hline CWO14 & $\begin{array}{l}\text { Capsicum } \\
\text { апnиum Linn. }\end{array}$ & 5 & 315 & $01.61 \%$ & Uniseriate & 25 & 300 & $07.69 \%$ & Uniseriate \\
\hline
\end{tabular}

Table 3: Stomatal Characteristics of some Members of Solanaceae in Parts of the Niger Delta Ecozone, Nigeria.

\begin{tabular}{|c|c|c|c|c|c|c|}
\hline $\begin{array}{l}\text { UPH. } \\
\text { Reference } \\
\text { No. }\end{array}$ & Name of Taxa & $\begin{array}{l}\text { Leaf } \\
\text { Surface }\end{array}$ & $\begin{array}{l}\quad \bar{x} \pm S . D \\
\text { Stomata } \\
\text { Length }(\mu \mathrm{m})\end{array}$ & $\begin{array}{l}\quad \bar{x} \pm S . D \\
\text { Stomata } \\
\text { Width }(\mu \mathrm{m})\end{array}$ & $\begin{array}{lr}\text { Coefficient } & \text { of } \\
\text { Variation for } \\
\text { Length of Stomata }\end{array}$ & $\begin{array}{lr}\text { Coefficient } & \text { of } \\
\text { Variation } & \text { for } \\
\text { Width } & \text { of } \\
\text { Stomata } & \\
\end{array}$ \\
\hline \multirow[t]{2}{*}{ CW001 } & \multirow{2}{*}{$\begin{array}{l}\text { Solanum } \\
\text { aethiopicum Linn. }\end{array}$} & Adaxial & $56 \pm 2.33$ & $36 \pm 1.76$ & $4.16 \%$ & $5.02 \%$ \\
\hline & & Abaxial & $54 \pm 1.72$ & $37 \pm 2.24$ & $3.19 \%$ & $6.09 \%$ \\
\hline \multirow[t]{2}{*}{ CW002 } & \multirow{2}{*}{$\begin{array}{l}\text { Solanum nigrum } \\
\text { Linn. }\end{array}$} & Adaxial & $43 \pm 1.61$ & $32 \pm 1.41$ & $3.74 \%$ & $4.39 \%$ \\
\hline & & Abaxial & $45 \pm 1.76$ & $24 \pm .7 .57$ & $3.96 \%$ & $31.54 \%$ \\
\hline \multirow[t]{2}{*}{ CW003 } & \multirow{2}{*}{$\begin{array}{ll}\text { Solanum torvum } \\
\text { Sw. }\end{array}$} & Adaxial & $55 \pm 1.76$ & $41 \pm 2.46$ & $3.20 \%$ & $6.00 \%$ \\
\hline & & Abaxial & $55 \pm 2.36$ & $36 \pm 1.72$ & $4.29 \%$ & $4.78 \%$ \\
\hline \multirow[t]{2}{*}{ CW004 } & \multirow{2}{*}{$\begin{array}{l}\text { Solanum } \\
\text { macrocarpon Linn. }\end{array}$} & Adaxial & $59 \pm 2.92$ & $38 \pm 3.06$ & $4.95 \%$ & $8.06 \%$ \\
\hline & & Abaxial & $57 \pm 2.74$ & $34 \pm 2.33$ & $4.81 \%$ & $6.86 \%$ \\
\hline \multirow[t]{2}{*}{ CW005 } & \multirow{2}{*}{$\begin{array}{l}\text { Solanum anomalum } \\
\text { Thonn. }\end{array}$} & Adaxial & $54 \pm 1.72$ & $35 \pm 1.76$ & $3.19 \%$ & $5.02 \%$ \\
\hline & & Abaxial & $54 \pm 1.72$ & $35 \pm 1.76$ & $3.19 \%$ & $5.02 \%$ \\
\hline \multirow[t]{2}{*}{ CW006 } & \multirow{2}{*}{$\begin{array}{l}\text { Solanum incanum } \\
\text { Linn. }\end{array}$} & Adaxial & $67 \pm 2.74$ & $39 \pm 2.92$ & $4.09 \%$ & $7.49 \%$ \\
\hline & & Abaxial & $59 \pm 2.46$ & $40 \pm 3.51$ & $4.17 \%$ & $8.789 \%$ \\
\hline \multirow[t]{2}{*}{ CW007 } & \multirow{2}{*}{$\begin{array}{l}\text { Solanum erianthum } \\
\text { D. Don. }\end{array}$} & Adaxial & $49 \pm 5.97$ & $20 \pm 1.02$ & $12.19 \%$ & $5.12 \%$ \\
\hline & & Abaxial & $52 \pm 2.11$ & $33 \pm 6.68$ & $4.05 \%$ & $2.02 \%$ \\
\hline \multirow[t]{2}{*}{ CW008 } & \multirow{2}{*}{$\begin{array}{l}\text { Solanum } \\
\text { lycopersicum Linn.. }\end{array}$} & Adaxial & $69 \pm 3.67$ & $42 \pm 3.44$ & $5.32 \%$ & $8.20 \%$ \\
\hline & & Abaxial & $71 \pm 4.83$ & $47 \pm 2.74$ & $6.80 \%$ & $5.84 \%$ \\
\hline \multirow[t]{2}{*}{ CW009 } & \multirow[t]{2}{*}{ Datura metel Linn. } & Adaxial & $136 \pm 10.75$ & $90 \pm 26.67$ & $29.63 \%$ & $12.60 \%$ \\
\hline & & Abaxial & $117 \pm 9.49$ & $87 \pm 9.49$ & $8.11 \%$ & $10.90 \%$ \\
\hline
\end{tabular}




\begin{tabular}{|c|c|c|c|c|c|c|}
\hline \multirow[t]{2}{*}{ CW010 } & \multirow{2}{*}{$\begin{array}{l}\text { Schwenckia } \\
\text { americana Linn. }\end{array}$} & Adaxial & $134 \pm 17.76$ & $89 \pm 14.49$ & $13.26 \%$ & $16.28 \%$ \\
\hline & & Abaxial & $130 \pm 25.82$ & $87 \pm 15.67$ & $19.86 \%$ & $18.01 \%$ \\
\hline \multirow[t]{2}{*}{ CW011 } & \multirow{2}{*}{$\begin{array}{l}\text { Physalis angulata } \\
\text { Linn. }\end{array}$} & Adaxial & $138 \pm 11.35$ & $84 \pm 11.74$ & $8.23 \%$ & $13.97 \%$ \\
\hline & & Abaxial & $103 \pm 29.08$ & $72 \pm 13.98$ & $28.23 \%$ & $19.42 \%$ \\
\hline CW012 & $\begin{array}{l}\text { Physalis micrantha } \\
\text { Linn. }\end{array}$ & $\begin{array}{l}\text { Adaxial } \\
\text { Abaxial }\end{array}$ & $\begin{array}{l}112 \pm 13.98 \\
83 \pm 11.60\end{array}$ & $\begin{array}{l}59 \pm 9.94 \\
50 \pm 8.17\end{array}$ & $\begin{array}{l}12.48 \% \\
13.98 \% .\end{array}$ & $\begin{array}{l}16.85 \% \\
16.34 \%\end{array}$ \\
\hline \multirow[t]{2}{*}{ CW013 } & \multirow{2}{*}{$\begin{array}{l}\text { Capsicum } \\
\text { frutescens Linn. }\end{array}$} & Adaxial & $133 \pm 11.40$ & $86 \pm 8.43$ & $8.72 \%$ & $9.81 \%$ \\
\hline & & Abaxial & $140 \pm 14.14$ & $77 \pm 8.23$ & $10.10 \%$ & $10.69 \%$ \\
\hline \multirow[t]{2}{*}{ CW014 } & \multirow{2}{*}{$\begin{array}{l}\text { Capsicum annuиm } \\
\text { Linn. }\end{array}$} & Adaxial & $127 \pm 31.98$ & $87 \pm 19.75$ & $25.18 \%$ & $21.71 \%$ \\
\hline & & Abaxial & $120 \pm 18.26$ & $76 \pm 12.65$ & $28.56 \%$ & $16.64 \%$ \\
\hline
\end{tabular}

Conclusion: In Solanaceae, stomatal and trichome complements are used to classify some species to a very great extent. There are both simple uniseriate and multiseriate trichomes. Contiguous cells, glandular trichomes, anomocytic and anisocytic stomata are very common in Solanaceae. Tetracytic and paracytic stomata are also observed in Solanaceae. Stomatal and trichome complements are vital diagnostic characters among species found in genera within any given family of plants. It is obvious that comparative studies among different families of plant may prove very difficult. It is not out of place that with the present day descriptions assigned in micro-morphological studies there may arise in the near future, stomatal and trichome classification that may be acceptable to an appreciable extent.

Acknowledgement: The authors acknowledge, with thanks, Prof. B. E. Okoli of the Department of Plant Science and Biotechnology, University of Port Harcourt, for his assistance.

\section{REFERENCES}

Arnold, E. (1973). Peacock's Elementary Micro technique. Pitman Press, Bath, Great Britain. Pp. 12-16.

Cutler, D. F. (1977). Applied Plant Anatomy Longman-Group Limited London.

Cutler, D. F. (1978). Applied Plant Anatomy .Lib. of Congr. Cataloguing in Publication Data. William Clowes and Sons Ltd London.

Metcalfe, C.R. and L. Chalk. (1979). Anatomy of the Dicotyledons. $2^{\text {nd }}$ eds. Vol. 1. The Clarendon Press. Oxford.

United States Department of Agriculture (2015): Solanum robustrum Natural Resource Conservation Service. PLANTS Database, USDA. Retrieved 17 November, 2015. 